\title{
Comparative Study of X-ray and High Resolution Computed Tomography in the Diagnosis of Interstitial Lung Disease
}

\author{
Tarak Patel ${ }^{1}$, Gurubharath Ilangovan ${ }^{2}$, Harshavardhan Balganesan ${ }^{3}$ \\ ${ }^{1}$ Junior resident, Department of Radiodiagnosis, Shri Sathya Sai Medical College and Research Institute, Chennai, Tamilnadu- \\ 603108, ${ }^{2}$ Professor and HOD, Department of Radiodiagnosis, Shri Sathya Sai Medical College and Research Institute, Chennai, \\ Tamilnadu- 603108, ${ }^{3}$ Senior resident, Department of Radiodiagnosis, Shri Sathya Sai Medical College and Research Institute, \\ Chennai, Tamilnadu- 603108, India
}

Corresponding author: Gurubharath Ilangovan, Professor and HOD, Department of Radiodiagnosis, Address: 100 A/5,11th sector,66th street,PonnambalamSalai KK nagar,Chennai,India 60078

DOI: http://dx.doi.org/10.21276/ijcmsr.2020.5.1.58

How to cite this article: Tarak Patel, Gurubharath Ilangovan, Harshavardhan Balganesan. Comparative study of $x$-ray and high resolution computed tomography in the diagnosis of interstitial lung disease. International Journal of Contemporary Medicine Surgery and Radiology. 2020;5(1):A263-A267.

\section{A B S T R A C T}

Introduction: Interstitial lung diseases (ILDs) are defined as a heterogeneous group of disorders that primarily affect the interstitium, a tissue of the alveolar wall between the capillary and the alveolar epithelium. These disorders can progress to irreversible pulmonary fibrosis and are important cause of morbidity and mortality. It is therefore imperative that a prompt and accurate diagnosis of the underlying cause is made so that appropriate management can be instituted.

Material and methods: This was a cross sectional study. Total 60 Patients, who referred from OPD of medicine and pulmonary medicine to the department of radio diagnosis, for diagnosis and evaluation of interstitial lung disease were included in the study and were subjected to chest radiograph followed by HRCT. Study duration was one and half years from Jan 2018 to June 2019. The results were tabulated, statistically analysed and compared with previous studies.

Result:The main result of the study was that HRCT was able to detect more abnormalities than conventional chest radiograp in the diagnosis of interstitial lung diseases. The differences between the two modalities were found to be statistically significant in certain findings.

Conclusion: Chest radiograph remain the basic and primary radiological tool for the diagnosis. HRCT is the best imaging modality and is an indispensable and crucial radiological tool in the diagnosis of ILD. As our understanding of these disorders advances and new therapeutic agents aimed at specific molecular targets are developed, it becomes increasingly important to achieve a specific diagnosis to facilitate the optimal management for patients with ILDs.

Keywords: Interstitial Lung Disease; HRCT; Connective Tissue Disease, Idiopathic Pulmonary Fibrosis, Usual Interstitial Pneumonia

\section{INTRODUCTION}

Interstitiallung diseases (ILDs) are defined as a heterogeneous group of disorders that affect the interstitium, which is a space between the capillary basement membranes. Infiltration, inflammation and fibrosis may affect the interstitial and the alveolar spaces at the same time. ${ }^{1}$ They are a diverse group of disorders of lung interstitium that are distinguished by acute and chronic inflammation and an irreversible process of fibrosis in alveolar walls and interstitium. The interstitium is defined as space between the alveolar epithelium and the capillary endothelium. This site is more prone for primary injury. "Interstitial" term can often be deceptive as it has additional effect on the capillaries and airway spaces, however its primary involvement is always on the interstitium only. ${ }^{2}$ The interstitial lung diseases are a diverse group of many respiratory tract disorders which will cause acute or chronic clinical illness. It is a common clinical problem which will affects individually as well as group also. Though they are grouped together, there are great variations in the risk factors for their development, the relevant changes, the associated prognosis, their pathological processes and making an accurate diagnosis is very essential. ${ }^{3}$

Recent studies and advantages like technological advances, notably physiologic testing, bronchoalveolar lavage, lung imaging studies, surgical lung biopsy and histopathological correlation improved our understanding towards these entities and for classifying it, as well as identification and understanding of the pathogenesis of ILD also. After arriving of high-resolution computed tomography (HRCT), approach to identify and diagnosis of usual interstitial pneumonia (UIP) from other idiopathic interstitial pneumonia is changed. it is also helpful to narrowed pathologic definition of usual interstitial pneumonia and recognition of prognostic importance. last the genetic medicine, the use of new technologies (e.g. mass spectrum analysis of proteins, microarrays and laser capture micro dissection) and also the development of animal models has 
had a significant impact on understanding the pathological process and potential molecular targets for interfering the fibro genesis. $^{4}$

Interstitial lung diseases are characterised by anatomical distortion of interstitium, alveolar epithelium and capillary endothelium, determined by a first stage of alveolitis followed by a stage of fibrosis. Interstitial lung diseases are characterised by slow and progressive destruction of alveolarcapillary cells, often it will lead to respiratory failure followed by death. For their smouldering evolution and non-specificity of symptoms like exertional dyspnoea and cough, they may remain undiagnosed and non-treated for a long time. For an early diagnosis of ILD the importance of HRCT and other investigations is well established.

Among all interstitial lung disease most common interstitial lung disease is idiopathic pulmonary fibrosis in adults and generally has a poor prognosis. ${ }^{3}$ Idiopathic pulmonary fibrosis (IPF)/usual interstitial pneumonia (UIP) is complicated and poorly-understood entity. Patients who are suffering from interstitial lung disease, approximately fifteen percentages of patients have underlying connective tissue disorders. ${ }^{5}$ Interstitial lung diseases also affect children in form of hypersensitivity pneumonitis and idiopathic interstitial pneumonia. ${ }^{6}$ Children who are suffering from gastro-oesophageal reflux, pulmonary hemosiderosis, lower respiratory tract infections (viral, bacterial and fungal), eosinophilic pneumonia, etc. may have an association with interstitial lung diseases. ${ }^{6-10}$

Among more than 100 entities of ILDs, a few of the disorders, including sarcoidosis, hemosiderosis, idiopathic pulmonary fibrosis and connective tissue disease related ILDs are the most common. ${ }^{11}$ Hence, adequate knowledge of these common entities is necessary in diagnosis and to rule out other differentials.

For the physician's diagnosis, characteristic signs of interstitial lung disease are diffuse or patchy pulmonary opacities on chest radiograph or a specific pattern of ILD on PFT. Diagnosis of ILD depends on various parameters like data on epidemics, clinico-radiological co-relation which will help in considering a diagnosis of high probability. ${ }^{12}$

There must be radiological, histopathological and clinical correlation for definitive diagnosis of ILD. Among all investigations the chest radiograph remains the basic and primary radiological tool for the diagnosis. However histologically proven 10-20\% ILD patients shows normal chest radiograph. ${ }^{13}$ Many respiratory diseases aren't diagnosed properly or stay hidden on chest radiograph, showing a "reticulonodular pattern" which is a nonspecific finding. ${ }^{2}$ It is not necessary that all different ILD's have similar radiological findings.

HRCT of the thorax is an indispensable and crucial radiological tool in the diagnosis of ILD. A competent diagnosis can usually be on the basis of HRCT findings associated with clinical context. ${ }^{11}$ Histopathological correlation with serologic testing might be useful in some cases. Recent advancements in HRCT technology has made imaging of lung parenchyma very efficient and effortless that too with excellent anatomical detail. ${ }^{14}$ The external features of scattered or diffuse parenchymal lung ailments is better expressed with high resolution. Compared to conventional chest radiography HRCT is more sensitive in the detection of ILDs. Even then, sensitivity isn't exactly $100 \% .{ }^{14}$ The specificity for the features of different lung parenchyma ailments has been recorded and appears to be better compared to conventional radiography. In clinical practice HRCT gives a big advantage to characterise and differentiate various types of ILD and to reach at a specific diagnosis.

In recent times, multiple research and reports have established that HRCT is highly sensitive and specific as compared to conventional chest radiography in effective diagnosis and determination of ILD. HRCT has repeatedly proved its effectiveness in the diagnosis of various respiratory disorders like IPF, silicosis, LAM, lymphangitic carcinomatosis, LIP and sarcoidosis. ${ }^{15}$

The findings of Mathieson et al are very important in establishing the importance and accuracy of HRCT and chest radiography in the specific diagnosis of patients with chronic diffuse ILD. Three observers interpreted chest radiographs and HRCT independently, noted the 3 potential diagnosis and appointed a degree of confidence. Highest confidence was achieved with $49 \%$ of HRCT and $23 \%$ of thorax radiographs and a pin-point diagnosis was reached with $93 \%$ and $77 \%$ respectively. In a large study, Grenier et al showed that HRCT was of high value when CT thorax was screened along with clinical and radiological findings. On the basis of clinical findings alone, an elaborate and accurate diagnosis was reached in $29 \%$ of the cases. When radiological and clinical findings were screened together it highly increased the optimism in the diagnosis to $54 \%$ and $80 \%$. Subsequently, many patients with a diagnosis of ILD on the basis of plain radiographs were forwarded for HRCT to establish a specific diagnosis or taper down the differential diagnosis. ${ }^{15}$ Nevertheless, only a limited amount of studies has integrated ILDs, chest radiographs and HRCT in one single study.

Study aimed to compare various patterns of interstitial lung disease in HRCT and in chest $\mathrm{x}$-ray with the objective to study various patterns of ILD in plain $x$-ray and in HRCT for the diagnosis of interstitial lung diseases and to study the distribution patterns of interstitial lung diseases

\section{MATERIALS AND METHODS}

This was a cross-sectional study, conducted in the department of radiodiagnosis, Shri Sathya Sai Medical College and Research Institute for a period of 18 months. Total 60 Patients, referred from OPD of medicine and pulmonary medicine to the department of radio diagnosis, SSSMCRI for diagnosis and evaluation of interstitial lung disease were subjected to conventional chest radiograph followed by HRCT. The institutional ethical clearance was obtained before initiation of study. informed consent was obtained from the concerned patients.

HRCT performed in a 16 SLICE GE CT SCAN machine in supine position. The window width was set between 10001300 and window level at -550 to -750 at $120 \mathrm{kVp}$ and 110 to $150 \mathrm{~mA}$. The pitch was set at $1: 1$

Patients also went for PA view of chest radiograph at $60 \mathrm{kVp}$ and $10-12.5 \mathrm{mAs}$ in a $500 \mathrm{~mA}$-ray machine 


\section{Inclusion criteria}

- Patients presented with dyspnoea and following chest $\mathrm{x}$-ray findings.

- Bilateral reticular or reticulonodular opacities.

- Unequivocal chest radiographs

- Honeycombing (end stage ILD)

- Poorly demarcated pleural-parenchymal borders along the hemi diaphragm and heart

- Distortion of lung architecture and changes of tractional bronchiectasis

- Ground glass abnormalities-increased attenuation of lung tissue without distortion of underlying blood vessels and bronchi

- Already diagnosed case of ILD

- Age group 20 year to 80 year

- Both sexes

\section{Exclusion criteria}

- Pregnant women

- Age group between 1 year to 20 year

- Known case of lung or cardiac anomalies like, CCF, tuberculosis etc

\section{STATISTICAL ANALYSIS}

Data entered in excel sheet, statistical analysis done by SPSS 23 software by using descriptive statistics and chi-square test

\section{RESULTS}

Detailed work up of all these patients was performed with the help of chest radiograph, HRCT chest scan, present clinical history, past and family history, occupational history and laboratory history.

Among all 60 patients, 27(45\%) patients were males and $33(55 \%)$ were females. In our study ILD were found more commonly at mean age of 46.73 years.

The spectrums of diseases included in the study were:

- $\operatorname{UIP}(57 \%)$

- $\quad \operatorname{NSIP}(18 \%)$

- $\operatorname{HP}(11 \%)$

- $\quad$ Sarcoidosis $(11 \%)$

- $\operatorname{ABPA}(3 \%)$

HRCT was more sensitive than radiography in detecting various imaging findings related to ILDs.

In our study reticular opacities were detected in $45(75 \%)$ cases by HRCT and in 30(50\%) $f$ cases by chest radiography. Similarly nodular opacities were detected in $45(75 \%)$ cases by HRCT and in 24(40\%) cases by chest radiography. Septal thickening was detected in 48(80\%) cases by HRCT and in $18(30 \%)$ cases by chest radiography.

Detection of honeycombing has great clinical significance for the diagnosis of ILDs. HRCT in our study detected honeycombing in 33(55\%) cases. Presence of honeycombing usually suggest end stage disease and hence HRCT proves to be an important modality for detection and timely management of patients with ILDs.

In our study, ground glass opacities, bronchiectasis, hilar and mediastinal lymphadenopathy were also detected in $33(55 \%)$ cases, 32(53\%) cases and 30(50\%) cases by HRCT

\begin{tabular}{|c|c|c|c|}
\hline SI. No. & Findings & Plain radiograph & HRCT \\
\hline 1 & Air space consolidation & 18 & 1 \\
\hline 2 & Septal thickening & 18 & 48 \\
\hline 3 & Reticular opacities & 30 & 45 \\
\hline 4 & Honeycombing & - & 33 \\
\hline 5 & Nodular opacities & 24 & 45 \\
\hline 6 & Distribution of nodules (perilymphatic, centrilobular or random) & NA & A \\
\hline 7 & Ground glass opacity & - & 33 \\
\hline 8 & Bronchiectasis & - & 32 \\
\hline 9 & Mosaic attenuation & - & 2 \\
\hline \multirow[t]{2}{*}{10} & Hilar and mediastinal lymphadenopathy & 9 & 30 \\
\hline & A-Applicable, NA-Not Applicable & & \\
\hline & Table-1: Comparative findings in $\mathrm{x}$ & Id HRCT & \\
\hline
\end{tabular}

\begin{tabular}{|c|c|c|c|c|c|c|c|}
\hline \multirow[t]{2}{*}{ Reticular opacity } & \multicolumn{2}{|c|}{ HRCT } & \multicolumn{2}{|c|}{ X RAY } & \multirow[t]{2}{*}{ Total } & \multirow[t]{2}{*}{$\mathrm{x}^{2}$} & \multirow[t]{2}{*}{ Total } \\
\hline & $\mathbf{N}$ & $\%$ & $\mathbf{N}$ & $\%$ & & & \\
\hline Present & 45 & $75 \%$ & 30 & $50 \%$ & 75 & \multirow[t]{3}{*}{8} & \multirow[t]{3}{*}{0.004} \\
\hline Absent & 15 & $25 \%$ & 30 & $50 \%$ & 45 & & \\
\hline Total & 60 & $100 \%$ & 60 & $100 \%$ & 120 & & \\
\hline
\end{tabular}

\begin{tabular}{|c|c|c|c|c|c|c|c|}
\hline \multirow[t]{2}{*}{ Nodular opacity } & \multicolumn{2}{|c|}{ HRCT } & \multicolumn{2}{|c|}{ X RAY } & \multirow[t]{2}{*}{ Total } & \multirow[t]{2}{*}{$\mathrm{X}^{2}$} & \multirow[t]{2}{*}{ Total } \\
\hline & $\mathbf{N}$ & $\%$ & $\mathbf{N}$ & $\%$ & & & \\
\hline Present & 45 & $75 \%$ & 24 & $40 \%$ & 69 & \multirow[t]{3}{*}{15.03} & \multirow[t]{3}{*}{0.0001} \\
\hline Absent & 15 & $25 \%$ & 36 & $60 \%$ & 51 & & \\
\hline Total & 60 & $100 \%$ & 60 & $100 \%$ & 120 & & \\
\hline
\end{tabular}




\begin{tabular}{|c|c|c|c|c|c|c|c|}
\hline \multirow[t]{2}{*}{ Septal Thickening } & \multicolumn{2}{|c|}{ HRCT } & \multicolumn{2}{|c|}{ Xray } & \multirow[t]{2}{*}{ Total } & \multirow[t]{2}{*}{$\mathrm{X}^{2}$} & \multirow[t]{2}{*}{ Total } \\
\hline & $\mathbf{N}$ & $\%$ & $\mathbf{N}$ & $\%$ & & & \\
\hline Present & 48 & $80 \%$ & 18 & $30 \%$ & 66 & \multirow[t]{3}{*}{30.00} & \multirow[t]{3}{*}{$<0.0000$} \\
\hline Absent & 12 & $20 \%$ & 42 & $70 \%$ & 54 & & \\
\hline Total & 60 & $100 \%$ & 60 & $100 \%$ & 120 & & \\
\hline
\end{tabular}

respectively. Mosaic attenuation was detected in 2(3\%) cases by HRCT (table-1).

The results were evaluated using descriptive statistical and chi square test. The significance value for chi square test was 0.05 .

Decision Criterion: we compare the $\mathrm{P}$ Value with the level of significance. If $\mathrm{P}<0.05$, we reject the null hypothesis and accept the alternate hypothesis. If $>0.05$, we accept the null hypothesis.

In our study HRCT detected reticular opacity in 45 (75\%) cases as compared to chest radiograph which detected reticular opacity in 30 (50\%) cases (table-2).

HRCT detected higher number of samples with reticular opacity compared to chest radiograph but the difference between the two methods was not statistically significant $(\mathrm{P}>0.05)$.

HRCT detected nodular opacity in 45 (75\%) cases as compared to chest radiograph which detected nodular opacity in 24 (40\%) cases (table-3).

HRCT detected higher number of samples with nodular opacity compared to chest radiograph. The difference between the two methods was found to be statistically significant $(\mathrm{P}<0.05)$.

HRCT detected septal thickening in 48 (80\%) cases as compared to chest radiograph which detected septal thickening only in 18 (30\%) cases (table-4).

HRCT detected higher number of samples with septal thickening compared to chest radiograph. The difference between the two methods was found to be statistically significant $(\mathrm{P}<0.05)$.

In our study Lower lobe and basal region of the lung is most commonly affected region detected on HRCT scan and most common type of involvement of interstitial lung disease is bilateral lung involvement. Bronchiectasis in 32 cases followed by lymphadenopathy in 30 cases were the most common associated findings in our study.

\section{DISCUSSION}

In our study higher number of patients with findings were detected by HRCT scan compared to plain chest radiograph. Chest radiograph also detected findings in our study which were significant but HRCT gave specific and accurate diagnosis in same patients. Therefore, HRCT was more accurate. HRCT also gave characteristic features at specific anatomy of lung parenchyma compared to chest radiograph. Some patients with clinical history, show normal chest radiograph. When we performed HRCT on those patients, they showed specific findings which were not there on chest radiograph. So, there is lack of sensitivity of conventional chest radiography in our study to diagnosis ILD's. In our study, 3 out of 60 patients had no findings in their chest radiographs, but HRCT showed focal radiological findings including thickened alveolar septa in these patients.

Reticular pattern was most common finding (50\%) seen on chest radiographs in our study. However, HRCT showed reticular patterns in $75 \%$ of the cases in our study, so sensitivity for reticular patterns are much greater by these both modalities in ILD.

In a study conducted by Martin Remy Jardin et $\mathrm{al}^{16}$ reticular opacity was detected by HRCT in $90 \%$ of all cases \& in $73 \%$ of cases by conventional chest radiography.

Another very common manifestation in ILD was nodular opacities in our study. 40\% patients showed nodular opacities in chest radiograph in our study. When we performed HRCT scan for nodular opacities, we got $75 \%$ patients who had nodular opacities. HRCT also gave characteristic feature about nodules such as their appearance, anatomical distribution and types like interstitial or air space nodules. Interstitial nodules have sharp margin compared to air space pulmonary nodule which is much appreciated by HRCT scan. $(\mathrm{P}<0.05)$.

In a study conducted by Martin Remy Jardin et al nodular opacity was detected by HRCT in $63 \%$ of all cases $\&$ in $37 \%$ of cases by conventional chest radiography and septal thickening was detected by HRCT in $50 \%$ of all cases \& in $20 \%$ of cases by conventional chest radiography.

HRCT can show evidence of ILD even when clinical and pulmonary function tests parameters are normal. ${ }^{17}$

By HRCT anatomical distribution of nodules were well appreciated. HRCT also helped to narrow down the differential diagnosis. Based on distribution of nodules on HRCT, they are classified into centrilobular, perilymphatic and random categories. Perilymphatic nodules which were common in sarcoidosis was well recognised in our study by HRCT scan. Similarly, in patients with history of military $\mathrm{TB}$ and viral infection which had random nodules in lung parenchyma was also well appreciated during HRCT Scan. From above all our study shows that HRCT scan has more specificity over conventional radiography.

\section{CONCLUSION}

Interstitial lung disease (ILD) is usually a delayed diagnosis because its clinical signs are often neglected and its respiratory symptoms are usually misdiagnosed to more common pulmonary diseases such as COPD. At the early stage, patients do require chest physician reference to diagnosis the ILD. HRCT scan is a choice of investigation to make diagnosis of ILD in their early stages. Early diagnosis and starting timely therapy in ILD patients are extremely helpful in view of improving the course of disease, its morbidity and mortality.

With the help of HRCT, diagnosis of ILD can be made 
easy as it helps in detecting abnormalities at the early stage with minimal clinical features or even when chest radiograph shows no significant abnormality.

In diagnosis of ILD chest radiograph is one of the primary and basic mode of investigation. HRCT scan of thorax is beneficial in patients with high clinical suspicion of ILDs and is also a key component of the multidisciplinary approach in diagnosis of ILDs. Different forms of ILDs are distinguished from one another by their specific pattern of abnormalities, and hence HRCT chest scans are needed for the diagnostic work up of ILD patients. HRCT scan helps in arriving to a conclusive diagnosis either alone or in conjunction with the clinical symptoms and other diagnostic modalities.

Many of the times, requirement for the lung biopsy can be eliminated with the help of specific and significant findings in HRCT.

\section{REFERENCES}

1. Neurohr C, Behr J. Dtsch Med Wochenschr.Diagnosis and therapy of interstitial lung diseases 2009; 134(11):524-9.

2. James Ward, Christine McDonald, et al. Interstitial lung disease: An approach to diagnosis and management. Australian Family Physician 2010:39(1);644-649.

3. Kevin K.Brown, M.D. Idiopathic Pulmonary Fibrosis: Current approach to diagnosis and therapy. MedSci update 2004; 21(5):1-3.

4. Crystal RG, Fulmer JD, Roberts WC, Moss ML, Line BR, Reynolds HY. Idiopathic pulmonary fibrosis: clinical, histologic, radiographic, physiologic, scintigraphic, cytologic and biochemical aspects. Ann InternMed 1976;85(3):769- 788 .

5. StrieterRM.Pathogenesis and natural history of usual interstitial pneumonia: the whole story or the last chapter of a long novel. Chest 2005;128(2):526S-532S.

6. Charlie Strange MD, Kristin B. Highland MD. Interstitial lung disease in the patient who has connective tissue disease. Clin Chest Med 2004;25(6): 549-559.

7. Vangveeravong $\mathrm{M}$, Schidlow DV. Interstitial lung diseases in children: a review.J Med Assoc Thai. 1995; 78(3):145-56

8. Bokulic RE, Hilman BC. Interstitial lung disease in children. PediatrClinNorth.Am. 1994;41(3):543-67.

9. Dinwiddie R, Sharief N, Crawford O. Idiopathic interstitial pneumonitis in children: a national survey in the United Kingdom and Ireland. PediatrPulmonol. 2002;34(1):23-29.

10. Fan LL, Kozinetz CA. Factors influencing survival in children with chronic interstitial lung disease. Am J RespirCrit Care Med. 1997;156(3 Pt 1):939-42.

11. Ryu JH, Daniels CE, Hartman TE, Yi ES. Diagnosis of interstitial lung diseases.MayoClin Proc. 2007; 82(8):976-86.

12. Valeyre D, Brauner M, Cadranel. Diagnostic approach in diffuse infiltrative lung diseases. J. Rev Prat. 2000; 50(17):1879-87.

13. David Sutton. Textbook of Radiology and Imaging Vol 1 Seventh edition, chapter 7, page 187.

14. Bessis L, Callard P, Gotheil C, et al. High resolution
CT of parenchymal lung disease: Precise correlation with histologic findings. Radiographics 1992;12(1):45.

15. Mayo JR, Webb WR, Gold R, et al. High resolution CT of the lung: an optimal approach. Radiology 1987;163(6):507-510.

16. Martin Remy-Jardin, M.D, Jacques Remy M.D., Catherine Deffontaines, M.D., Alain Duhamel,PhD. Assessment of diffuse infiltrative lung disease: comparison of conventional CT and high-resolution CT. Radiology October 1991;181(2):157- 162.

17. S Raniga, P Sharma, G Kaur, A Arora, Y Khalasi, PA Vohra. Interstitial lung disease in rheumatoid arthritis- a study of thirty cases. Ind J RadiolImag 2006;16(4):835839.

\section{Source of Support: Nil; Conflict of Interest: None}

Submitted: 15-02-2020; Accepted: 28-02-2020; Published online: 23-03-2020 